\title{
Enhancing community based health programs in Iran: a multi-objective location-allocation model
}

\author{
S. Khodaparasti ${ }^{1}$ H. R. Maleki ${ }^{2}$ - S. Jahedi ${ }^{2}$ - M. E. Bruni ${ }^{3}$ - P. Beraldi ${ }^{3}$
}

Received: 22 July 2015 / Accepted: 6 April 2016 / Published online: 26 April 2016

(c) Springer Science+Business Media New York 2016

\begin{abstract}
Community Based Organizations (CBOs) are important health system stakeholders with the mission of addressing the social and economic needs of individuals and groups in a defined geographic area, usually no larger than a county. The access and success efforts of CBOs vary, depending on the integration between health care providers and CBOs but also in relation to the community participation level. To achieve widespread results, it is important to carefully design an efficient network which can serve as a bridge between the community and the health care system. This study addresses this challenge through a locationallocation model that deals with the hierarchical nature of the system explicitly. To reflect social welfare concerns of
\end{abstract}

\author{
S. Khodaparasti \\ khodaparasti.sara@yahoo.com \\ H. R. Maleki \\ maleki@sutech.ac.ir \\ S. Jahedi \\ jahedi@sutech.ac.ir \\ M. E. Bruni \\ mariaelena.bruni@unical.it \\ P. Beraldi \\ patrizia.beraldi@unical.it
}

1 Department of Mathematics and Computer Science, University of Calabria, Cosenza, Rende, Italy

2 Faculty of Mathematics, Shiraz University of Technology, Shiraz, Iran

3 Department of Mechanical, Energy and Management Engineering, University of Calabria, Cosenza, Rende, Italy equity, local accessibility, and efficiency, we develop the model in a multi-objective framework, capturing the ambiguity in the decision makers' aspiration levels through a fuzzy goal programming approach. This study reports the findings for the real case of Shiraz city, Fars province, Iran, obtained by a thorough analysis of the results.

Keywords Community based organization · Location theory $\cdot$ Hierarchical facility system $\cdot$ Multi-objective programming $\cdot$ Data envelopment analysis

\section{Introduction}

Over the past decades, major sociological and economic changes, such as the growing population and the increased urbanization, have placed increasing pressure on the Iranian health system $[9,19,20]$. In this context, the role of the health care system has been challenged [20], fostering considerable transformations. One of the most visible breakthroughs has been the establishment of a Primary Health Care network that has led to remarkable achievements in various areas, ranging from health education to endemic disease control $[19,20]$. To reduce the gap between health outcomes in urban and rural areas, given the shortage of human and capital resources, the Primary Health Care system has relied on the community participation.

Locally-based groups, referred to as CBOs, which have operated in Iran for centuries, are playing an increasingly important role in social and economic developments, strengthening the foundations of an emergent civil society. In the case of Iran, CBOs are active in meeting and coping with the critical needs of underserved and vulnerable 
population. They promote community health and education, provide counseling services, drug addiction prevention, and improve service delivery, thereby explicitly reflecting social welfare concerns of equity and local accessibility.

Whether effective and equitable development can be ultimately achieved by CBOs depends on the community participation they foster, its main determinant being the geographical proximity [3].

The aim of the paper is to explore the potential for a structured quantitative approach for the strategic location of CBOs, considering the geographical distribution of specialized health care services.

The challenges addressed in this paper are manifold. From a modeling viewpoint, it investigates the optimal location of CBOs, proposing a novel multi-objective hierarchical location-allocation model. To the best of our knowledge, this is the first attempt to develop a mathematical model for this problem. Moreover, the model contributes to the location-allocation literature, since it combines the multi-objective and the hierarchical paradigms, thereby providing a faithful representation of the system and facing the concerns of the different stakeholders involved.

From a practical viewpoint, the insights derived from the research provide a systematic analysis of the trade-offs in the above mentioned applicative domain and shed light on determinant factors affecting the system efficiency and equity.

The first phase of this project consisted in a field-based research to better understand the system from an operational perspective. In this respect, for carrying out data collection, we collaborated with CBOs of Shiraz city, Fars Province, Iran. This collaboration with the practitioners was an essential component of this research rooted in a real-life application.

The paper is organized as follows. In Section 2, the relevant literature is briefly reviewed. In Section 3, the characteristics of the system, the nature of the services provided, and the stakeholders' goals are discussed. A mathematical programming model for the specific case study is then presented. In Section 4, a solution methodology for solving the proposed model is described. Section 5 is devoted to the discussion of the findings and the policy implications suggested by the results. Finally, in Section 6, some conclusions are drawn.

\section{Literature review}

This paper presents a new multi-objective hierarchical location-allocation model for a health care network. To place the contribution of the paper in the right perspective, we restrict our focus to the literature on location-allocation and multi-objective models applied in health care.

\subsection{Location-allocation models}

There is a vast literature on the application of locationallocation models in the health care sector [25]. Syam and Corte in [36] presented a location-allocation model for specialized health care services, providing treatment and rehabilitation for strokes or traumatic brain injuries. The model minimizes the total cost, taking into account the effects of factors such as service centralization, facility overload costs, and target utilization levels. In [4], Benneyan et al. introduced a location-allocation model for long-term decision makings in Veterans Health Administration sector, considering the fluctuation in demands. The objective function is a weighted sum of conflicting criteria including travel time, unoccupied capacity, and uncovered demands. In another paper, Zhang et al. [41] investigated the impact of client choice behavior in the location of preventive care facilities. The main aim is the maximization of the participation level which is proportional to the geographical proximity. In [14], a location-allocation model for the design of a primary health care network is presented. Three criteria, including the maximization of the coverage, the participation, and the total traveled distance are separately considered as accessibility measures. Song et al. [31] introduced a new location-allocation model for the design of long-term health care services where the preferences of the patients are incorporated through closest assignment constraints. Kim and Kim [17] proposed a location-allocation model for locating new public health care services in a network of existing private and public facilities. The model deals with the preferences of low-income and high-income patients as well as the competition level among private and public facilities.

Following the hierarchical stream in location-allocation models, Galvao et al. studied the problem of locating perinatal facilities in Rio de Janeiro, where a nested hierarchy structure between different types of facilities exists [11, 12]. In [21], Mestre et al. presented a hierarchical model in which the improvement in the geographic equity of access is followed by the minimization of weighted distance traveled by the users within a hierarchical multi-service health care system. The study incorporates the efficiency of service as well as the operational costs. Sahin et al. proposed a 2-level hierarchical location model for locating facilities which provides blood services in Turkish Red Crescent [27]. In [29], Smith et al. presented a hierarchical location model for locating community health facilities in developing countries. 


\subsection{Multi-objective models in health care sector}

The importance of the simultaneous consideration of multiple objective functions has been acknowledged in some of the location-allocation models reviewed in Section 2.1 $[4,14]$. Recognizing the compelling necessity of considering different criteria, Mohammadi et al. [24] proposed a bi-objective location-allocation model for the design of a reliable health care network. The first objective minimizes the total cost of treatment, transportation, and the expected cost of failure, while the second objective minimizes the sum of maximal accumulated travel time. In [34], Sun et al. presented a bi-objective allocation model for the optimal assignment of patients to hospitals during pandemic influenza outbreak. The two considered criteria are the minimization of the total distance traveled by patients to hospitals as well as the minimization of the maximum distance traveled by a patient to the assigned hospital. In [1], a multi-objective mathematical model for the allocation of beds to hospitals with uncertain demands has been presented. The model investigates the trade-off between three criteria: the cost of creating new beds, the number of nurses and physicians. In a recent research, Steiner et al. presented a multi-objective model to aggregate the health services offered in different municipalities into some microregions. The aggregation is done to facilitate the management of resources [32]. The model provides a trade-off between three conflicting criteria, including maximizing the variety of services provided in each microregion, minimizing the inter-microregion travel distances, and maximizing the homogeneity of population in the microregions.

In [15], Guo et al. presented a bi-objective locationallocation model for the evaluation of community based health services. The model investigates a trade-off between cost and service where service is expressed as the total number of demand nodes that receive service within a given distance threshold. In another research, Mitropoulos et al. [23] developed a three-objective location model to find the most effective locations for locating health centers in Greece. The model investigates the trade-off between the total distance traveled by customers to their closest hospital, the underachievement in the minimum workload requirement of hospitals, and the average Data Envelopment Analysis (DEA) scores assigned to open hospitals. In [10], Davari et al. presented a bi-objective model for health care design in which the equity criterion has been considered. They also implemented fuzzy goal programming approach to solve the model. The model addresses the trade-off between two criteria, including the aggregate participation level in the network and the equity. To this end, they incorporate the attractiveness concept as a negative exponential function of travel time or distance. The participation criterion is evaluated by maximizing the total attractiveness captured by served demands, and equity criterion is expressed by the maximization of the minimum attractiveness in the network. In another work, Graber-Naidich et al. [13] presented a three-objective location-allocation model to account for cost, accessibility, and appropriateness of provided care for a primary care network design problem. In [5], Cardoso et al. presented a location-allocation model addressing longterm care network design in which three types of equity are considered, including equity of access, geographical equity, and socioeconomic equity. Syam and Cote [35] developed a location-allocation model for establishing not-for-profit health care organizations. As the authors mention, the proposed model is an implicitly multi-objective model in which the objective function takes into account the cost of service and the penalty cost for the total unmet demands. The model also requires that a minimum service level be provided.

A hierarchical multi-objective location model for the design of hospital networks has been proposed by Mestre et al. [22]. The model considers the uncertainty in demands. In [30], Smith et al. introduced a bi-objective hierarchical location-allocation model with equity and efficiency criteria. The model is especially focused on public health services. Recently, another approach balancing equity and efficiency has been provided with an application to emergency service design [16].

Despite the quite rich literature reviewed, there are potential gaps and open issues yet to be investigated. Despite the large number of applications for location-allocation models in the health care field, there are only a few papers addressing the hierarchical nature of health care services [11, 12, 21, 22, 27, 29], two of which, [22, 29], incorporate the multi-objective framework. Moreover, our multi-service hierarchical location model incorporates the preferences of referred recipients to upper level facilities. To this end, referral is only limited to a subset of upper level facilities which are within a specified threshold. This will facilitate the referral and encourage the recipients to continue their treatment which, in turn, will increase the level of participation. We recognized that except equity and accessibility, which have already been addressed [22, 29], the efficiency of existing upper level facilities is another important factor affecting the system performance. This issue is of paramount importance especially in hierarchical systems, where the quality of professional services provided at upper levels has a direct influence on the final outcome. The incorporation of efficiency helps the managers to recognize the most efficient facilities that deserve more financial support. On the other hand, considering the competition level component is 
another contribution of the present paper. The model tries to decrease the competition level among first level facilities by increasing the distance between any pair of those facilities. This gives servers located at optimal locations more chance to develop their service and to attract more recipients in a stable condition.

Another important issue usually neglected or only partially addressed in the health care literature is the incorporation of ambiguity in different stakeholders' preferences at different levels. To overcome this shortcoming, we have considered the fuzzy set theory that enables the Decision Maker (DM) to express the preferences imprecisely and even as linguistic terms.

\section{Problem description and formulation}

The State Welfare Organization in Iran coordinates three different service providers: CBOs, Consulting Centers (CCs), and Addiction Treatment Clinics (ATCs). CBOs are small organizations with different headquarters in the city. The services provided by CBOs can be classified into two main components: basic consulting services and addiction prevention programs. Other services provided by CBOs are somehow related to these two types of services. They also hold workshops teaching life and work skills. The establishment of CBOs is essential for linking the district office and the local community. As a matter of fact, in recent years, CBOs have been successful in decreasing crime and addiction rates in poor neighborhoods $[2,26]$. As a result, the local district supported the establishment of CBOs, technically and financially, providing human resources such as social workers and consultants. It should be mentioned that the CBOs neither interfere in addiction treatment nor provide the patients with methadone. In addition, they do not give the recipients professional consulting services. Instead, CBOs refer a portion of their recipients to other higher level facilities where they can receive more professional and cut-rate services, based on the needs and the severity of recipients' problems. In particular, professional CCs (both public and private) offer consulting services in different fields such as personal, family, child care and development as well as educational problems. They work under the supervision of district authorities that require accountability. ATCs work under either the supervision of district authorities or medical universities, and they offer inpatient and outpatient addiction treatment services.

The nature of this organization imposes a hierarchical structure into the system where CBOs are at the lowest level and the CCs and the ATCs provide service at the highest level. The service providers operate under a successively exclusive service hierarchy. This means that any upper level facility provides just its own level of service. In fact, although ATCs and CCs can also offer lower level services, they do not do so in most cases. It should be noted that both CCs and ATCs have their own recipients, and the recipients referred by CBOs consist of only a portion of their customers.

The structure of the system is explained in Fig. 1. Note that level 0 denotes customers.

Many factors should be considered when designing a health care system of this kind, where the participation and completion of treatment rates are of paramount importance. The activity level of a facility is determined by the number of people who choose to seek its services. Therefore, service areas of the facilities cannot be neglected in the
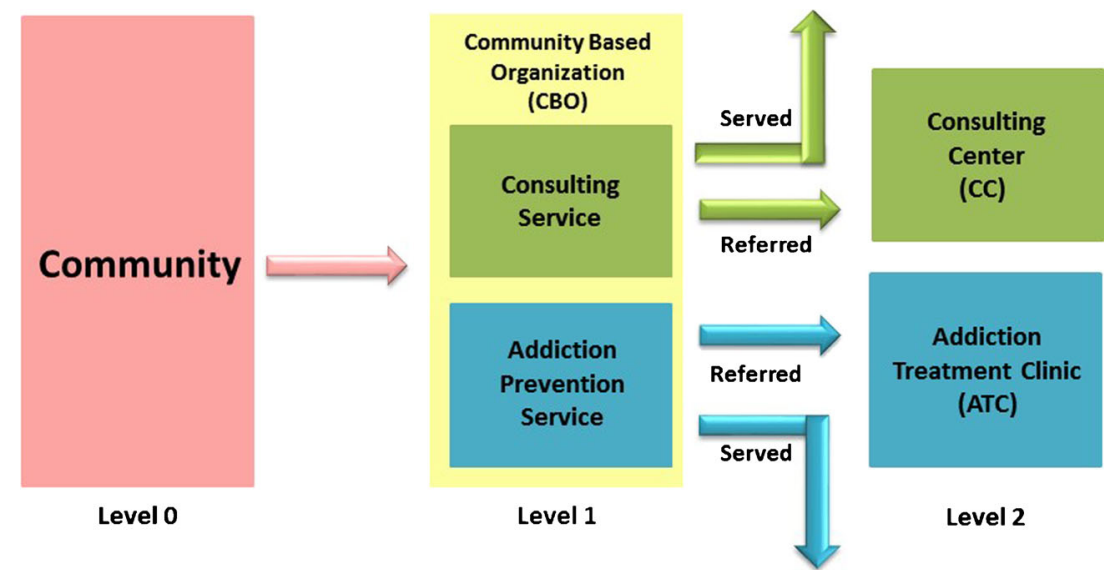

Level 2

Fig. 1 The system structure 
design process. One of the major determinants of participation to the programs offered by CBOs is the ease of access to that facility. The factors that impact the accessibility include the number, type, and location of the facilities. As well recognized [23, 39], the probability of participation and the motivation for continuing treatment decrease with the distance traveled. As a matter of fact, many patients stop their addiction treatment or do not attend their consulting sessions because of long distance.

In order to facilitate the accessibility of patients to the second-level facilities, the total distance traveled by recipients referred to the second-level facilities should be minimized. In addition, the referral is limited only to those second-level facilities which are within a critical distance. To improve the performance of the service network, some other criteria should be taken into account. For example, to improve the service level of any first-level facility and to increase system safety, first-level facilities should be dispersed throughout the city, avoiding strenuous competition levels. This implements an equity concern since it ensures equal access to CBOs to people living in different areas. In addition, the maximum distance between first- and second- level facilities should be minimized to assure that the second-level facilities are well dispersed over the area.

Besides this spatial efficiency, another concern of the stakeholders is the facility efficiency, expressed as a fraction of outputs produced for a given level of inputs. In this way, economical concerns, important especially in this context where the providers operate with limited resources, are also considered.

To address the efficiency and productivity of sited facilities, we apply the DEA framework, as a well-known tool for quantitative efficiency assessment which is widely applied in the operations research field. For more information about the DEA, the interested reader is referred to $[8,23]$.

\subsection{The mathematical model}

The mathematical model for the design of the system is presented in this section. We start by introducing the notation used throughout the article, and then present the objective functions and the constraints.

\section{Sets/Indices}

$I: \quad$ is the set of demand points indexed by $i$

$F:$ is the set of potential locations to site first-level facilities indexed by $f$
$F(T) \subseteq F:$ denotes set of first-level facilities such that $d_{i f} \leq T$, where $\mathrm{T}$ is the maximum threshold distance that recipients should travel to reach first-level facilities

$K:$ is the set of service types (addiction treatment or specialized consulting) provided by second-level facilities indexed by $k$

$S_{k}:$ is the set of potential locations to site second-level facilities providing service type $k$

$S=\cup_{k \in K} S_{k}: \quad$ is the set of locations to site second-level facilities indexed by $s$

$S_{k}(\hat{T}) \in S_{k}: \quad$ set of the second-level facilities providing service $k$ such that $d_{f s} \leq \hat{T}$ where $\hat{T}$ is the maximum threshold distance that recipients referred from a first-level facility should travel to reach a second-level facility.

\section{Input parameters}

$h_{i}: \quad$ denotes the amount of demand at demand point $i, i \in I$

$d_{a, b}:$ denotes the distance between two arbitrary points or locations $a$ and $b$

$\beta_{i}^{k}$ : denotes the percentage of recipients from demand point $i$ requiring service type $k$ at the second-level

$c:$ denotes the capacity (the maximum number of demand nodes) of the first-level facility $f$

$C^{k}$ : denotes the capacity of the second-level facility $s$ providing service $k$

$\eta_{s}$ : denotes the amount of inefficiency associated with the second-level facility $s$

$p: \quad$ denotes the maximum number of first-level facilities

$q^{k}:$ denotes the maximum number of second-level facilities providing service type $k$

$M$ : denotes the maximum distance between first-level facilities $\left(M=\max _{f, f^{\prime} \in F} d_{f f^{\prime}}\right)$

$\mathrm{m}\left(m=\min _{f \in F, s \in S} d_{f s}\right):$ denotes the minimum distance between first- and second-level facilities

\section{Decision variables}

$y_{i f}^{1}=\left\{\begin{array}{l}1, \text { if demand node } i \text { is allocated to the first-level facility } f \\ 0, \text { otherwise }\end{array}\right.$ $x_{f}^{1}=\left\{\begin{array}{l}1, \text { if a first-level facility is established at potential site } f \\ 0, \text { otherwise }\end{array}\right.$

$x_{s}^{2}= \begin{cases}1, & \text { if a second-level facility providing service } k \text { is established } \\ & \text { at potential site } s \\ 0, & \text { otherwise }\end{cases}$ $w_{f s}^{12}=$ the amount of demand referred from the first-level facility $f$ to the second-level facility $s$

$D_{1}$ : the minimum distance between any pair of first-level facilities

$D_{12}^{k}$ : the maximum distance between any pair of firstlevel and second-level facilities offering service $k$. 
The model reads as follows.

$$
\begin{aligned}
& \min O_{1}(\mathbf{w})= \sum_{f \in F} \sum_{k \in K} \sum_{s \in S_{k}} d_{f s} w_{f s}^{12} \\
& \min O_{2}(\mathbf{x})= \sum_{k \in K} \sum_{s \in S_{k}} \eta_{s} x_{s}^{2} \\
& \min O_{3}(\mathbf{D})= \sum_{k \in K} D_{12}^{k}-D_{1} \\
& \sum_{f \in F(T)} y_{i f}^{1}=1 \quad \forall i \in I \\
& \sum_{i \in I} y_{i f}^{1} \leq c x_{f}^{1} \quad \forall f \in F \\
& \sum_{f \in F} w_{f s}^{12} \leq C^{k} x_{s}^{2} \quad \forall k \in K, \forall s \in S_{k} \\
& \sum_{s \in S_{k}(\hat{T})} w_{f s}^{12}-\sum \sum_{i \in I} \beta_{i}^{k} h_{i} y_{i f}^{1}=0 \\
& D_{12}^{k}+\left(x_{f}^{1}+x_{s}^{2}\right)\left(m-d_{f s}\right) \geq 2 m-d_{f s} \\
& \forall f \in F, \forall k \in K, \forall s \in S_{k} \\
& D_{1}+\left(x_{f}^{1}+x_{f^{\prime}}^{1}\right)\left(M-d_{f f^{\prime}}\right) \leq 2 M-d_{f f^{\prime}} \\
& \forall f, f^{\prime} \in F\left(f \neq f^{\prime}\right) \\
& \sum_{f \in F} x_{f}^{1} \leq p \\
& \sum_{s \in S_{k}} x_{s}^{2} \leq q^{k} \quad \forall k \in K \\
& x_{f}^{1}, x_{s}^{2} \in\{0,1\} \quad \forall f \in F, \forall s \in S \\
& y_{i f}^{1} \in\{0,1\} \quad \forall i \in I, \forall f \in F \\
& w_{f s}^{12} \geq 0 \quad \forall f \in F, \forall k \in K, \forall s \in S_{k} \\
& D_{12}^{k} \geq 0 \quad \forall k \in K \\
& D_{1} \geq 0
\end{aligned}
$$

Departing from the rationale explained at the beginning of this section, the three objectives of accessibility, efficiency, and equity are operationalized into three different objective functions. The accessibility is represented by $O_{1}$ where the total travel time for individuals accessing secondlevel facilities, once referred from CBOs, is minimized. This assures spatial efficiency and maximizes at the same time the success probability of the program or the participation level.

The efficiency criterion is represented by $\mathrm{O}_{2}$, which minimizes the total inefficiency score of second-level facilities in the system. The equity is represented by $O_{3}$. It includes two components: the maximum distance between any pair of open facilities offering similar services but at different levels, $D_{12}^{k}$, and the minimum distance between any pair of open CBOs, $D_{1}$.

Constraints (4) state that recipients should be referred only to CBOs within a critical distance. Constraints (5) impose a limit on the number of quarters that any $\mathrm{CBO}$ can serve. Constraints (6) impose a limit on the number of referral from any open CBO to the upper level facilities. The next restrictions in (7) are associated with service referrals. They enforce recipients to be referred to second-level facilities which are within a critical distance. Constraints (8) define distance variable $D_{12}^{k}, \forall k \in K$, as the maximum distance between any pair of open facilities offering similar services at different levels. These restrictions in combination with (3) provide the minimum dispersion between any pair of lower and upper open facilities. Constraints (9) define dispersion variable $D_{1}$ as the minimum distance between any pair of first-level facilities. In a similar way, constraints (9) in combination with (3) assure that open CBOs are as much as possible dispersed throughout the area. Constraints (10) impose a limit on the number of first-level facilities to be opened. The set of constraints in (11) put an upper bound on the total number of upper level facilities to be established. Finally, constraints (12)-(16) define the type of variables.

In the next section, we will describe an approach for dealing with the multi-objective nature of the proposed model.

\section{The fuzzy goal programming approach}

For solving a multi-objective problem, a wide variety of multi-objective programming techniques can be applied. These methods differ from each other with respect to their different ways of converting the multi-objective model into a single objective one [33].

The goal programming approach is one of the most popular and practical approaches used [6]. In the goal programming framework, the DM imposes an aspiration (target) level on each criterion. Then, the deviations from these target levels are minimized. The most challenging issue in goal programming is the determination of aspiration levels as precise values. In fact, the main question to answer here is: how can the DM set precise values for targets, especially at the presence of some degrees of uncertainty which is inevitable in most real-world problems? To capture the ambiguity in the aspiration levels, the fuzzy set theory can be used [42]. The resulting method, known as fuzzy goal programming approach, enables the DM to express the target levels imprecisely and even as linguistic terms such as "approximately greater (less) than" or "approximately equal to". 
In this case study, we apply the weighted additive model introduced by Tiwari et al. [38] and also the priority preemptive approach developed by Chen and Tsai [7]. Both these models guarantee that the obtained solution is a Pareto efficient one.

Based on the main idea behind fuzzy goal programming approach, the mathematical model in (1)-(16) can be expressed as the problem of finding a feasible solution for the set of constraints in Eqs. 17-18, as follows:

$$
\begin{aligned}
& O_{h}(.) \preceq O_{h}^{I} \quad h=1,2,3 \\
& (1)-(16)
\end{aligned}
$$

where $O_{h}^{I}$ represents the aspiration levels assigned with fuzzy goal in Eq. $17 h=1,2,3$. Note that $\preceq$ denotes the fuzziness in the fuzzy goal and can be interpreted as "approximately less than or equal to". Fuzzy goals in Eq. 17 represent fuzzy sets and can be identified by their membership functions indicating the degree of utility in achieving the target values.

The linear membership functions assigned to fuzzy goals in Eq. 17 are defined as follows:

$\mu_{O_{h}}()=. \begin{cases}1 & O_{h}(.) \leq O_{h}^{I} \\ 1-\frac{O_{h}(.)-O_{h}^{I}}{O_{h}^{N}-O_{h}^{I}} & O_{h}^{I} \leq O_{h}(.) \leq O_{h}^{N} \\ 0 & O_{h}(.) \geq O_{h}^{N}\end{cases}$

where $O_{h}^{I}$ and $O_{h}^{N}$ are, respectively, the aspiration level (ideal solution) and the upper bound (nadir solution) assigned to fuzzy goal $\mu_{O_{h}}(),. h=1,2,3$.

To obtain the ideal solution for the $h^{\text {th }}$ criterion, we solve a single objective problem including criterion $O_{h}($.) and the set of constraints in (1)-(16). The nadir solution, for the $h^{\text {th }}$ criterion, is the maximum value that $O_{h}$ (.) takes with respect to the ideal solutions of the other two criteria.

Applying the weighted additive model (Tiwari et al. [38]), an equivalent formulation for the initial fuzzy model in (17)-(18) is obtained as follows:

$$
\begin{aligned}
\max & \sum_{h=1}^{3} \omega_{h} \mu_{O_{h}}(.) \\
& (1)-(16) \text { and }(19) \\
& \mu_{O_{h}}(.) \leq 1 \quad h=1,2,3 \\
& \mu_{O_{h}}(.) \geq 0 \quad h=1,2,3
\end{aligned}
$$

where $\omega_{h}\left(\omega_{h}>0, \sum_{h=1}^{3} \omega_{h}=1\right)$ denotes the weight associated with the $h^{\text {th }}$ fuzzy goal.

In multi-objective context, very often, the DM considers a priority structure in which some fuzzy goals have a higher priority for the achievement over the others and the fuzzy goals are ranked into different priority levels. Following this stream, Chen and Tsai [7] proposed the preemptive priority fuzzy goal programming model capturing the priorities imposed over goals. For our special case, the managers have considered two different priority levels for the fuzzy goals. The fuzzy goal associated with the criterion $O_{1}$ is ranked as the 1 -priority, whereas the fuzzy goals associated with criteria $\mathrm{O}_{2}$ and $\mathrm{O}_{3}$ are ranked as the 2-priority. The preemptive priority model can be formulated as follows:

$$
\begin{aligned}
\max & \sum_{h=1}^{3} \mu_{O_{h}}(.) \\
& \mu_{O_{2}}(.) \leq \mu_{O_{1}}(.) \\
& \mu_{O_{3}}(.) \leq \mu_{O_{1}}(.) \\
& (1)-(16) \text { and }(19) \\
& \mu_{O_{h}}(.) \leq 1 \quad h=1,2,3 \\
& \mu_{O_{h}}(.) \geq 0 \quad h=1,2,3
\end{aligned}
$$

The algorithmic scheme of the presented fuzzy goal programming approach is reported below.

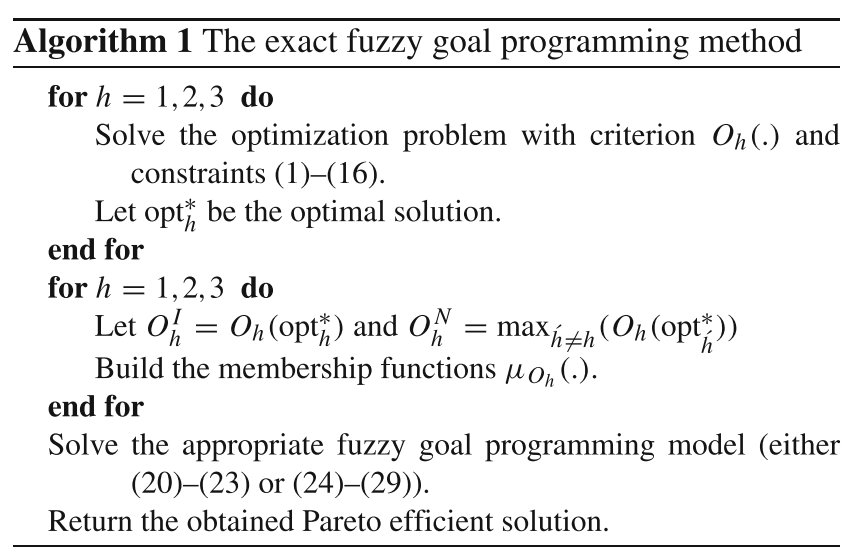

Theorem 1 The solution produced by the Algorithm 1 is a Pareto efficient solution for the proposed model.

Proof Let $Z^{*}$ be the optimal solution of Eqs. 20-23, where $Z^{*}$ denotes the set of optimal variables $\left(\mathbf{x}^{*}, \mathbf{y}^{*}, \mathbf{w}^{*}, \mathbf{D}^{*}\right)$. If $Z^{*}$ is not an efficient solution for the model in (1)-(16), there exists another feasible solution $Z$ such that $O_{h}(Z) \leq$ $O_{h}\left(Z^{*}\right) h \in\{1,2,3\}$ and for some $j \in\{1,2,3\}$ we have $O_{j}(Z)<O_{j}\left(Z^{*}\right)$. This results in $\mu_{O_{h}}(Z) \geq \mu_{O_{h}}\left(Z^{*}\right) h \in$ $\{1,2,3\}$ and $\mu_{O_{j}}(Z)>\mu_{O_{j}}\left(Z^{*}\right)$ for some $j \in\{1,2,3\}$. Since $\omega_{h}$ are strictly positive values, we conclude that $\sum_{h=1}^{3} \omega_{h} \mu_{O_{h}}(Z)>\sum_{h=1}^{3} \omega_{h} \mu_{O_{h}}\left(Z^{*}\right)$ which is a contradiction with the optimality of $Z^{*}$. The same results hold for the preemptive priority model. 


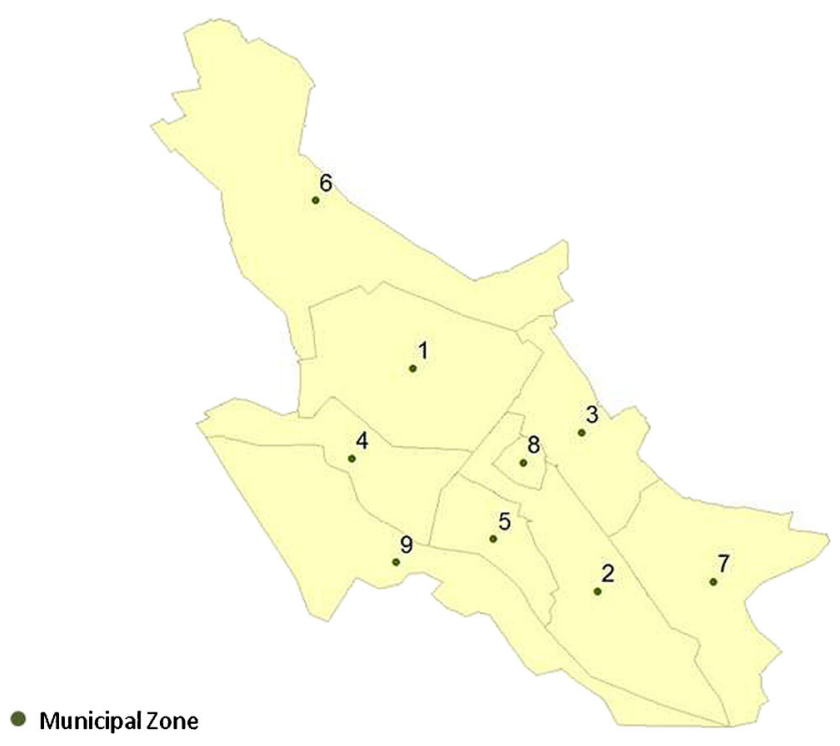

Fig. 29 Municipal zones of Shiraz city

\section{Case study}

As the sixth most populous city in Iran and the capital of Fars province, Shiraz city is well-known for being an important historical, cultural, political, economical, and commercial center. The specific geographical and economical characteristics of Shiraz city and its potential have encouraged many people to choose this city for immigration. Based on the national census of population and housing reports, prepared by statistical center of Iran in 2011, the population of the municipality of Shiraz is about 1700687 people and in the last 5 years, 175081 people have immigrated to this city.

The social structure of the municipality of Shiraz is also very complex due to the immigrants of different socioeconomic backgrounds from other parts of the country, especially from rural areas and small cities in Fars province. This fact resulted in higher population density and increased exposure to deteriorated living conditions.

Most immigrants, coming from small cities and rural parts, are settled in suburban, poor, and crime-prone neighborhoods. The unemployment dilemma, addiction and drugrelated crimes are serious concerns threatening the immigrant community. As a matter of fact, Fars province is among the first 12 provinces having the highest addiction prevalence rate [43]. In this situation, the main aim of the district authorities is to provide the residents with appropriate social care services, including addiction prevention and treatment programs as well as consulting services for improving life quality. The municipality of Shiraz includes nine municipal zones which are depicted in Fig. 2.
To choose both the set of demand points and candidate facility locations, we concentrate on the municipal zones associated with higher crime rates. In 2010, Taghvaii et al. conducted a research to evaluate the crime rate in eight existing municipal zones of Shiraz [37]. Based on their findings, zone 2 reports the highest rate for drug-related crimes with $42.6 \%$, followed by zones 5,7 , and 8 with $14.6 \%$. The rate of drug-related crimes in other zones varies between $1.1 \%$ to $4.4 \%$. Zone 9 is not surveyed in the research because it has been recently included in municipal zone divisions. Nevertheless, we consider it as a crime-prone area with a rather high rate of immigration located at the outskirts of the city. We discretized the municipal area in 84 population centers, representing the demand nodes.

Currently, 24 CBOs, most of which located in the crimeprone areas with a high rate of immigration, provide lower level services concerning consulting programs and addiction prevention plans. Besides the 24 existing locations, we have considered 20 new locations in order to investigate the potential system improvement. Although some existing CBOs in municipal zones 1 and 6 are included in our study, we did not consider any new candidate $\mathrm{CBO}$ or demand node in these areas. The reason is that some CBOs in these areas did not have any considerable experience of referring their recipients to upper level facilities.

At present, 30 active CCs, including 29 private centers and a public one, are run under the supervision of the local authority. Currently, 10 ATCs, including 9 private clinics and a public one, provide addiction treatment services. Besides these 10 locations, we have considered 8 extra potential sites for establishing new ATCs.

Figure 3 illustrates the spatial representation of demand nodes as well as both existing and potential sites for locating first-level and second-level facilities performed by ArcMap. We assume that at most $35 \mathrm{CBOs}, 14 \mathrm{CCs}$, and 13 ATCs can be sited, i.e., $p=35, q_{1}=14$ and $q_{2}=13$.

To evaluate the efficiency score of each CC, we have considered the input and output parameters of Table 1. Then, the Klimberg and Ratick's simultaneous DEA model (see [18]) has been used to compute the DEA scores. The efficiency of ATCs has not been considered in our study given the limited number of existing ATCs.

At first sight by looking at Fig. 3, it seems that CCs have not been sited in proper locations. For instance, we noticed that 17 out of 30 existing CCs are located in municipal zone 1 , where demand for CBO services is considerably low. In other words, $57 \%$ of CCs are not well spread out over the city. We also found that the rate of referral from existing CBOs to some CCs is very low.

In order to instantiate the model, we have considered a coverage distance for CBOs equal to 2.5 kilometers to facil- 


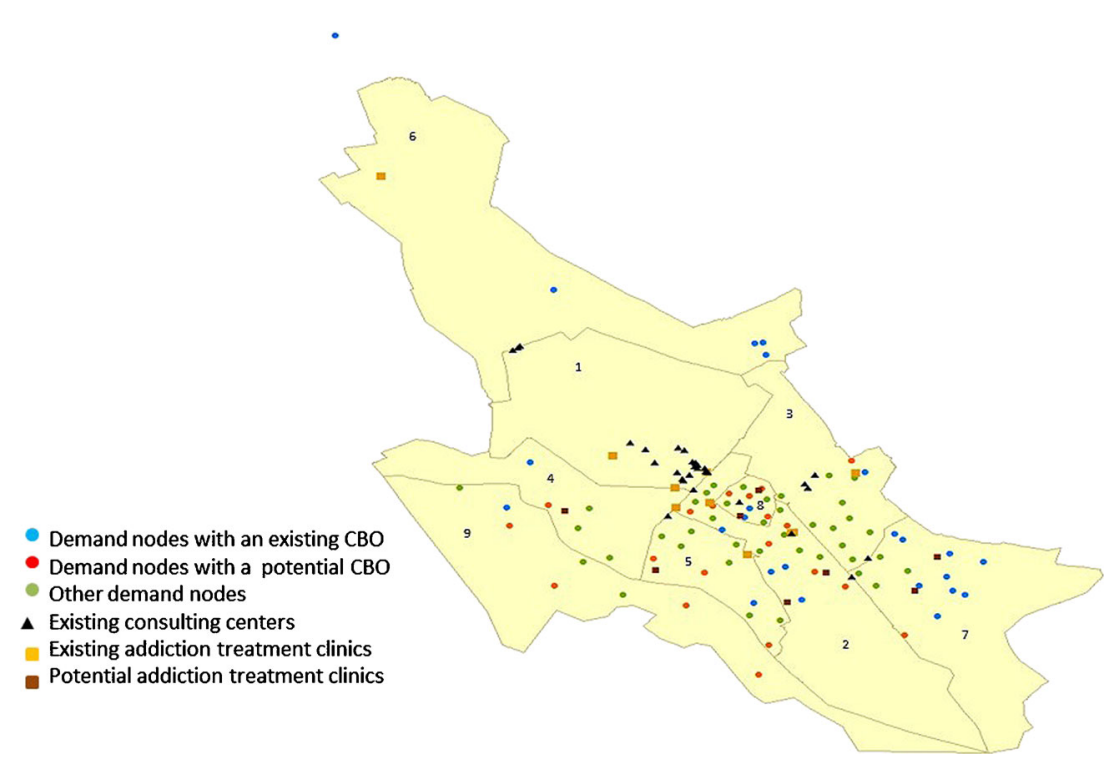

Fig. 3 Spatial distribution of population centers and existing and potential locations of facilities

itate the residences' access and encourage them to interact with CBOs constantly. The appropriate coverage distance threshold for the second-level facilities has been set to 4.5 and 6 kilometers for CCs and ATCs, respectively.

To gather the needed information about the rate of referral from existing CBOs to upper level facilities and the number of demands at each demand point, we asked CBO managers to fill in our questionnaire. Other data about CBOs and ATCs were collected by contacting the managers of the local authorities. Collecting data as well as modeling the case study began in May 2013 and ended in March 2014.

\subsection{Presentation of the results}

This section is devoted to the presentation of the results obtained by solving the optimization problem for the case of Shiraz. The optimization problem was modeled using AIMMS 4.1 and solved by CPLEX 12.6, running on a 2.7

Table 1 Input and output parameters for CCs

\begin{tabular}{ll}
\hline Inputs & Outputs \\
\hline $\begin{array}{l}\text { Number of doctors } \\
\text { Number of psychology } \\
\text { consultants }\end{array}$ & $\begin{array}{c}\text { Number of non-referred treated recipients } \\
\text { by CBOs }\end{array}$ \\
Maximum capacity & \\
\hline
\end{tabular}

MHz personal laptop with 4 G RAM. The CPU time for all cases did not deviate from 3 seconds.

We present three research questions, relevant for the DMs of Shiraz, hereafter called scenarios $A, B$, and $C$. To provide the managers with alternative solutions and to compare the current system with the solutions obtained by the model, we amended the problem (1)-(16) with the following constraint:

$$
\left|J_{e}\right|-\sum_{f \in J_{e}} x_{f}^{1} \leq q_{e}
$$

where $J_{e}$ denotes the set of existing CBOs' locations and $q_{e}$ is the maximum number of existing CBOs to be closed. Hence, $\left|J_{e}\right|-\sum_{f \in J_{e}} x_{f}^{1}$ represents the number of existing CBOs which are closed and this number is limited above by $q_{e}$.

\subsection{The current system}

In this subsection, we evaluate the current network based on the three main criteria, including accessibility, facility efficiency, and equity.

Looking at Fig. 3, we observe that 9 out of 24 existing CBOs (the $38 \%$ of first-level facilities) have been located at municipal zone 7 , while there are only 4 active CBOs (the $17 \%$ of first-level facilities) at municipal zone 2 , which has the highest rate for drug-related crimes. In addition, municipal zones 5 and 8 have the same rate for drug-related crimes as municipal zone 7, but they are hosting only 1 and 2 active CBOs, respectively. This shows that the current spatial con- 
figuration of the first-level facilities is not consistent with the demands at different areas.

Moreover, the current location of CBOs is not balanced. As a matter of fact, the minimum distance among all pairs of the 24 existing CBOs is only $0.29 \mathrm{~km}$, indicating a high competition level among CBOs.

Considering the current configuration of CBOs and the limitation on their capacities, the managers are able to cover only $52 \%$ of demand nodes within the threshold of 2.5 $\mathrm{km}$. The percentage of uncovered demand nodes is around $44 \%$ of the total demands in the network. The minimum distance traveled by these recipients to their closest CBO is 4.72 kilometers, whereas the average is 3.69 kilometers. This is an evidence for the fact that, using existing CBOs, it is impossible to provide service for all quarters.

The location of uncovered demand nodes and their neighborhoods can guide the managers to find appropriate potential sites for establishing new facilities. There is no complete information about the referral pattern which is used in the system. However, by gathering the data, especially in the case of $\mathrm{CCs}$, we noticed that some recipients are referred to $\mathrm{CCs}$, which are very far from their closest CBO. Even supposing the ideal case in which recipients are referred to their closest second-level facilities, the distance traveled to reach their closest $\mathrm{CC}$ and ATC is 6.14 and 8.48 kilometers, respectively.

In the rest of this section, we investigate the optimal configuration of facilities under three different scenarios, referred to Case $A, B$, and $C$, that provide meaningful answers to a specific questions posed by the managers. For each case, a comparison with both the current system and other cases are provided.

\subsection{Case A}

This case answers the question: how should the current network be reorganized?

In other words, the managers adopt a risk-prone approach accepting the consequences of closing some of the existing CBOs in order to get the best possible spatial configuration

Table 2 The optimal results for case A

\begin{tabular}{lllllll}
\hline & $O_{1}$ & $O_{2}$ & $O_{3}$ & $\mu_{1}$ & $\mu_{2}$ & $\mu_{3}$ \\
\hline$O_{1}^{*}$ & 5890.540 & 7.670 & 68.394 & 1 & 0 & 0 \\
$O_{2}^{*}$ & 11518.775 & 2.721 & 67.559 & 0.11 & 1 & 0.25 \\
$O_{3}^{*}$ & 12237.342 & 4.730 & 65.090 & 0 & 0.59 & 1 \\
FGP & 6998.799 & 2.827 & 65.350 & 0.83 & 0.98 & 0.92 \\
\hline
\end{tabular}

for the whole system. In this case, the corresponding model is exactly the same as the model in (1)-(16).

First of all, the solution obtained by the fuzzy goal programming method is compared with the solutions obtained by considering separately the three objective functions. The results are shown in Table 2. The first three rows in Table 2 represent the optimal objective function values resulting from the solution of single objective problems with criteria $O_{1}, O_{2}$, and $O_{3}$, respectively while the last row shows the results for the fuzzy goal programming model, denoted by FGP.

Columns 5-7, in Table 2, show the utility degree in achieving the target value for each criterion.

The results show that, when $O_{1}$ is optimized, the highest utility degree for the accessibility criterion $\left(\mu_{1}=1\right)$ is achieved at the price of sacrificing the other two criteria completely $\left(\mu_{2}=\mu_{3}=0\right)$. Similarly, the utility degree associated with the efficiency criterion is at the highest level $\left(\mu_{2}=1\right)$, while for the other two criteria this value is low ( $\mu_{1}=0.11, \mu_{3}=0.25$ ) when $O_{2}$ is optimized.

Similar conclusions can be drawn for the third row. Unlike the first three cases, the optimal solution of the fuzzy goal programming model provides significantly higher utility degrees in target achievement for all the criteria.

The optimal spatial configuration for first-level and second-level facilities obtained by the fuzzy goal programming method is shown in Fig. 4. In addition, Table 6 in Appendix reports the open facilities for both the lower and the upper levels.

Comparing the configuration with the current one, we observe that in the former case the minimum distance between any pair of open CBOs is 0.48 , whereas in the latter is 0.29 kilometers. It is interesting to note that although 11 extra CBOs are established, to provide extra coverage, the minimum distance between CBOs increases by $65 \%$. This finding shows that, for this special case study, a higher number of CBOs does not exacerbate necessarily the competition level between them.

Hence, within an optimal configuration of CBOs, we are able to provide coverage for all demand nodes and at the same time to decrease the competition level between CBOs. By relocating $\frac{1}{3}$ of existing $\mathrm{CBOs,}$, the system reaches higher utility degrees for both accessibility and equity criteria.

Moreover, the results show that existing ATCs 4 and 9 are not included in the optimal solution and only 14 out of $30 \mathrm{CCs}$ are selected. We should emphasize that, since $50 \%$ of active $\mathrm{CCs}$ in the new configuration are efficient, the recipients have more chance to be appropriately served than before. 
- Existing CBO

- Newly established CBO

- Selected CC

- Existing ATC

- Newly established ATC

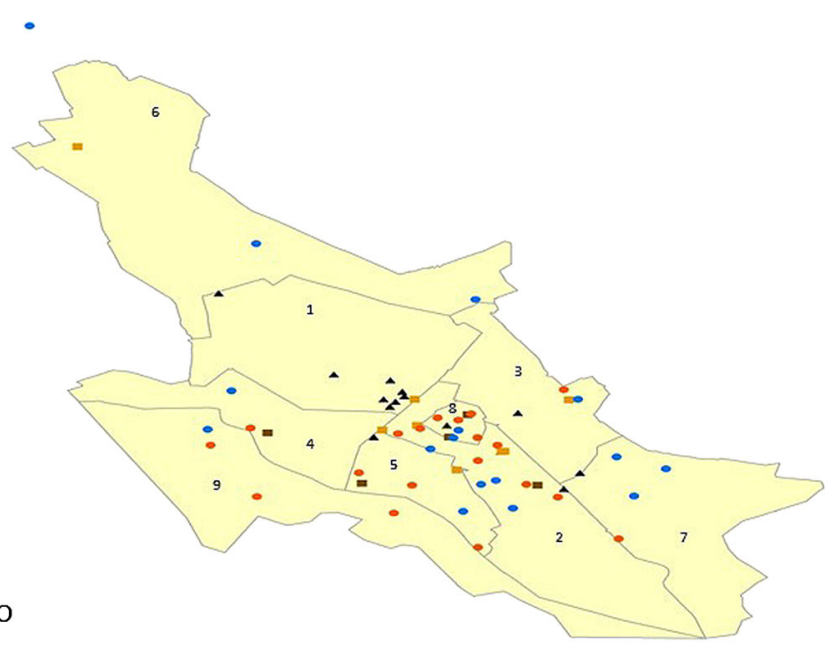

Fig. 4 Spatial distribution of optimal locations for case $A$

In addition, the maximum distance between any pair of CBOs and CCs and ATCs, linked with referrals, are 4.340 and 5.80 kilometers, respectively. The average of the aforementioned distance for CCs (ATCs) is 2.953 (2.419). Comparing with the current system, this shows about $0.29 \%$ (20\%) decrease in the maximum distance traveled by the referred demands to their assigned CCs (ATCs).

\subsection{Case B}

This case is relevant when the managers adopt a risk averse policy for the first-level facilities. In particular, this scenario let the DMs know how the current network could be upgraded without closing existing CBOs and establishing 11 new CBOs to provide coverage for the under-serviced areas.

The model associated with case $B$ is (1)-(16) amended with the set of constraints $x_{f}^{1}=1, \quad f \in J_{e}$ to preserve the existing CBOs. Table 3 shows the objective function values

Table 3 The optimal results for case B

\begin{tabular}{lllllll}
\hline & $O_{1}$ & $O_{2}$ & $O_{3}$ & $\mu_{1}$ & $\mu_{2}$ & $\mu_{3}$ \\
\hline$O_{1}^{*}$ & 6929.891 & 7.670 & 68.394 & 1 & 0 & 0 \\
$O_{2}^{*}$ & 11518.775 & 2.721 & 67.559 & 0.04 & 1 & 0 \\
$O_{3}^{*}$ & 12237.342 & 4.730 & 65.090 & 0 & 0.56 & 1 \\
FGP & 8324.161 & 2.827 & 65.541 & 0.73 & 0.98 & 1 \\
\hline
\end{tabular}

for single objective problems as well as the results for the fuzzy goal programming model.

Conclusions similar to the previous case can be drawn from Table 2.

Using the multi-objective approach, the total utility degree assigned to all criteria is significantly higher than the total utility achieved for each single objective problem.

Comparing the solutions with the case $A$, we can notice a deterioration of both accessibility and equity criteria in the case $B$. In particular, the deterioration in the accessibility criterion is about $19 \%$ while the equity criterion is slightly $(0.3 \%)$ worse.

The deterioration is related to the fact that case $A$ has more flexibility in finding the best configuration of firstlevel facilities. The value of the facility efficiency criterion is the same in both cases. This is an expected behavior, since the policy taken for the establishment of first-level facilities does not affect the efficiency of the second-level facilities (CCs).

To investigate the possibility of improving the accessibility criterion, we apply the preemptive priority model

Table 4 The optimal results for case $B$ with priority structure

\begin{tabular}{lllllll}
\hline & $O_{1}$ & $O_{2}$ & $O_{3}$ & $\mu_{1}$ & $\mu_{2}$ & $\mu_{3}$ \\
\hline PPFGP & 7789.475 & 10.859 & 66.128 & 0.83 & 0.92 & 0.79
\end{tabular}




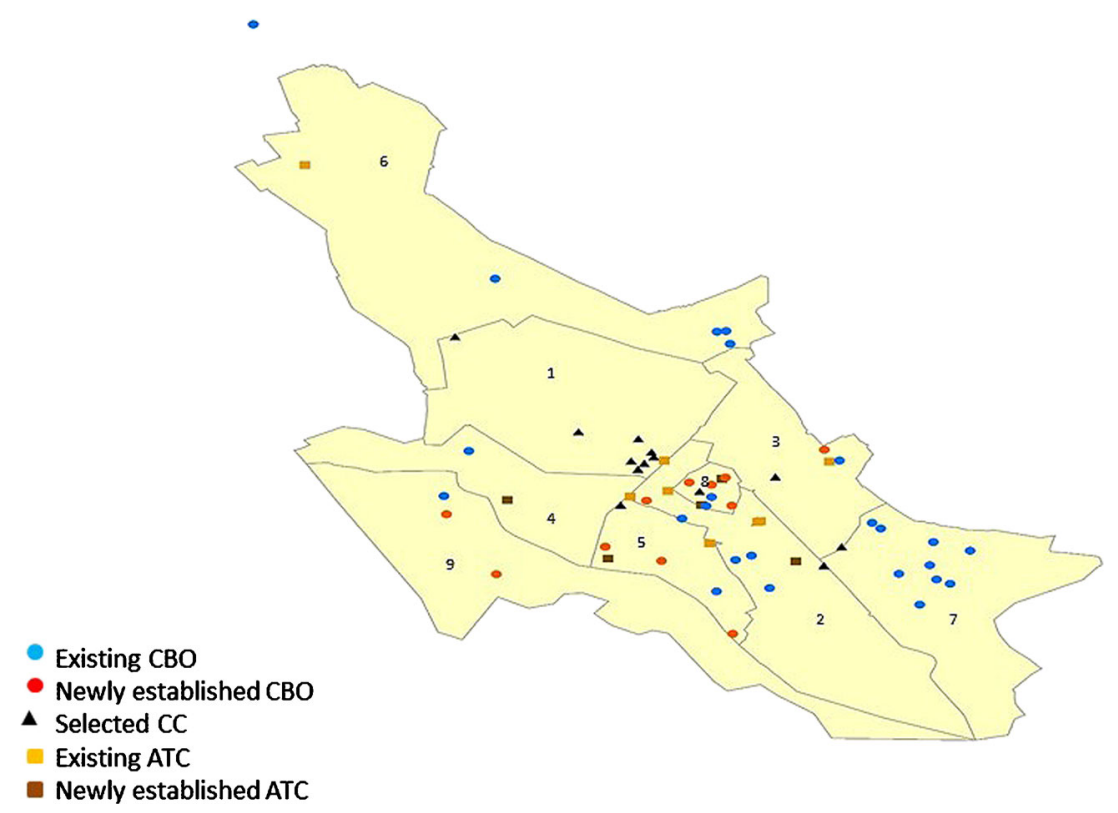

Fig. 5 Spatial distribution of optimal locations for case $B$

(24)-(29) considering the case that the accessibility criterion is prioritized over both facility efficiency and equity criteria.

The results of the preemptive priority model are summarized in Table 4, where PPFGP denotes the preemptive priority fuzzy goal programming model.

The results show that an improvement of $6.4 \%$ in the accessibility criterion can be obtained at the price of deteriorating facility efficiency $(2.8 \%)$ and equity criteria $(0.9 \%)$.

The spatial distribution of the optimal locations is shown in Fig. 5. See also Table 8 in the Appendix.

Comparing the optimized configuration with the current configuration we can notice that 8 out of 10 of the existing ATCs are located at optimal locations. This can guide the managers to relocate ATCs positioned in non-optimal locations.

Table 5 The optimal results for case C

\begin{tabular}{lllllll}
\hline & $O_{1}$ & $O_{2}$ & $O_{3}$ & $\mu_{1}$ & $\mu_{2}$ & $\mu_{3}$ \\
\hline$O_{1}^{*}$ & 6025.212 & 8.141 & 68.394 & 1 & 0 & 0 \\
$O_{2}^{*}$ & 11618.733 & 2.721 & 68.394 & 0.02 & 1 & 0 \\
$O_{3}^{*}$ & 11749.450 & 5.02 & 65.264 & 0 & 0.58 & 1 \\
FGP & 7190.792 & 2.827 & 65.350 & 0.80 & 0.98 & 0.97 \\
\hline
\end{tabular}

\subsection{Case C}

Another relevant question is whether the current network could be upgraded while limiting the number of existing CBOs to be closed. The results have been obtained by solving the model (1)-(16) amended with the constraint (30), allowing at most 4 existing CBOs to be closed.

The spatial distribution of the optimal locations is shown in Fig. 6. See also Table 8 in Appendix.

Table 5 indicates the optimal objective function values for the fuzzy goal programming problem. Comparing the results of the last row in Table 5, with similar results in Table 3, we can draw the following conclusions:

In terms of accessibility, this case provides better results than the case $B$, with an increase of $14 \%$. The equity criterion is also improved, since the minimum distance between any pair open CBOs is $65 \%$ higher. As far as the efficiency criterion is concerned, both cases provide the same results.

On the other hand, the case $A$ provides higher accessibility levels ( $2.7 \%$ higher) than case $C$, whilst both cases provide the same results for the other two criteria.

\subsection{Discussion}

The analysis of these different planning contexts suggested important managerial insights. First of all, they showed that 


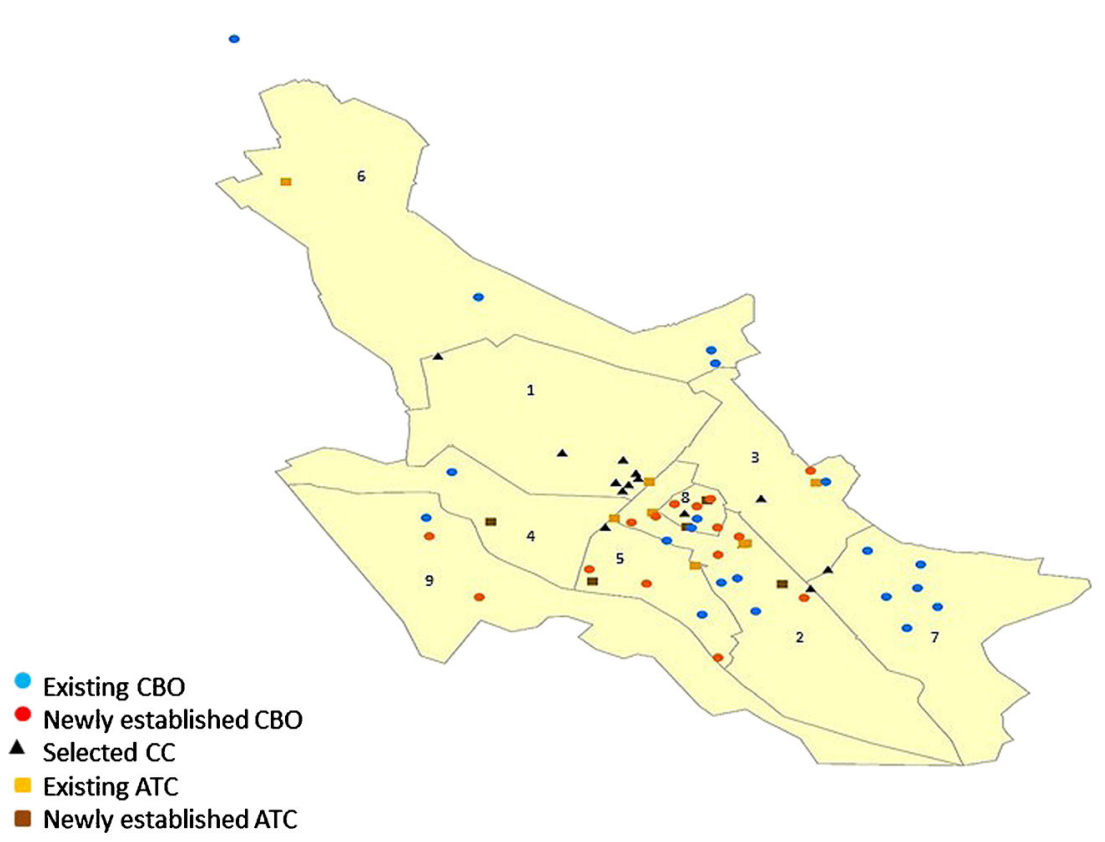

Fig. 6 Spatial distribution of optimal locations for case $C$

the existing CBOs are far from being able to meet the community needs, leaving many areas without access.

Regarding the accessibility criterion, case $A$ provides the best results. This is expected as, in this case, the managers accept the risk of closing some existing CBOs. The model uniformly distributes the resources across the city, thereby ensuring the provision of services to the highest number of individuals.

Regarding the equity criterion, the maximum distance between the first-level and second-level facilities for all cases are the same and the only difference is in the minimum distance between CBOs. In this respect, cases $A$ and $C$ provide better values than the case $B$. This, on one hand, underlines a high competition level between CBOs in the current system and, on the other hand, it highlights the importance of adopting quantitative approaches in the configuration of these services.

In addition, case $A$ has the lowest value for the average distance traveled by recipients to reach ATCs $(32 \%$ and $12 \%$ improvement over cases $B$ and $C$, respectively), whereas in the current system, both the total weighted distance traveled to the second-level facilities and the average distance traveled to reach CCs/ATCs are high.

All the cases provide the same results for the efficiency criterion. This result is reasonable as this criterion is independent from the policy adopted for the selection of CBOs.
It is remarkable that, for all cases, all efficient CCs are included in the optimal solutions.

\section{Conclusions}

In this paper, a new combined multi-objective hierarchical location model arising in the context of community based services has been proposed. The model accounts for three different policy objectives: equity, accessibility, and efficiency. The notion of ambiguity in the decision makers desiderata' has been taken into account, underlying the importance of the human component in the optimization phase. This is a salient model feature for the DMs, who were able to express the objectives in linguistic terms. In addition, the investigation of the trade-off between these criteria enabled the managers to explore the structure of the system in detail and take decisions in which both the managers' perspectives and public viewpoints are considered. The proposed model was validated using real data for Shiraz city in Iran. By implementing the solution of the mathematical model, the managers could practically evaluate the improvement in the completion of therapy sessions and addiction treatments, especially for this case study.

The model built is quite general and can easily be adapted to different contexts with similar characteristics, 
although it was inspired by the situation of Shiraz city. As a future research deserving detailed examination, we mention the incorporation of qualitative measures in a comprehensive model. It would be interesting to see the trade-off between qualitative and quantitative measures for performance assessment.

Another interesting avenue for future research is the application of the VIKOR method to find the compromise solution that better suits the DM's preferences $[28,40]$.

Acknowledgments The authors gratefully acknowledge Iran Drug Control Headquarters and the local State Welfare Organization in Shiraz for providing us with their data.

\section{Appendix}

Table 6 Optimal locations for case A

\begin{tabular}{rrrrrrrrrrrrr}
\hline$x_{f}^{1}$ & $x_{f}^{1}$ & $x_{f}^{1}$ & $x_{f}^{1}$ & $x_{f}^{1}$ & $x_{f}^{1}$ & $x_{f}^{1}$ & $x_{1 s}^{2}$ & $x_{1 s}^{2}$ & $x_{1 s}^{2}$ & $x_{2 s}^{2}$ & $x_{2 s}^{2}$ & $x_{2 s}^{2}$ \\
\hline 1 & 9 & 18 & 23 & 29 & 34 & 40 & 1 & 10 & 20 & 1 & 7 & 15 \\
3 & 10 & 19 & 25 & 30 & 35 & 41 & 4 & 12 & 27 & 2 & 8 & 17 \\
4 & 11 & 20 & 26 & 31 & 36 & 42 & 5 & 14 & 29 & 3 & 10 & 18 \\
5 & 13 & 21 & 27 & 32 & 37 & 43 & 7 & 16 & 30 & 5 & 13 & - \\
7 & 15 & 22 & 28 & 33 & 38 & 44 & 9 & 17 & - & 6 & 14 & - \\
\hline
\end{tabular}

Table 7 Optimal locations for case B

\begin{tabular}{rrrrrrrrrrrrr}
\hline$x_{f}^{1}$ & $x_{f}^{1}$ & $x_{f}^{1}$ & $x_{f}^{1}$ & $x_{f}^{1}$ & $x_{f}^{1}$ & $x_{f}^{1}$ & $x_{1 s}^{2}$ & $x_{1 s}^{2}$ & $x_{1 s}^{2}$ & $x_{2 s}^{2}$ & $x_{2 s}^{2}$ & $x_{2 s}^{2}$ \\
\hline 1 & 6 & 11 & 16 & 21 & 33 & 38 & 1 & 10 & 20 & 1 & 7 & 15 \\
2 & 7 & 12 & 17 & 22 & 34 & 40 & 4 & 12 & 27 & 2 & 8 & 17 \\
3 & 8 & 13 & 18 & 23 & 35 & 41 & 5 & 14 & 29 & 3 & 10 & 18 \\
4 & 9 & 14 & 19 & 24 & 36 & 42 & 7 & 16 & 30 & 5 & 13 & - \\
5 & 10 & 15 & 20 & 32 & 37 & 43 & 9 & 17 & - & 6 & 14 & - \\
\hline
\end{tabular}

Table 8 Optimal locations for case C

\begin{tabular}{rrrrrrrrrrrrr}
\hline$x_{f}^{1}$ & $x_{f}^{1}$ & $x_{f}^{1}$ & $x_{f}^{1}$ & $x_{f}^{1}$ & $x_{f}^{1}$ & $x_{f}^{1}$ & $x_{1 s}^{2}$ & $x_{1 s}^{2}$ & $x_{1 s}^{2}$ & $x_{2 s}^{2}$ & $x_{2 s}^{2}$ & $x_{2 s}^{2}$ \\
\hline 1 & 7 & 13 & 20 & 27 & 33 & 38 & 1 & 10 & 20 & 1 & 7 & 15 \\
3 & 8 & 15 & 21 & 28 & 34 & 40 & 4 & 12 & 27 & 2 & 8 & 17 \\
4 & 9 & 17 & 22 & 30 & 35 & 41 & 5 & 14 & 29 & 3 & 10 & 18 \\
5 & 10 & 18 & 23 & 31 & 36 & 42 & 7 & 16 & 30 & 5 & 13 & - \\
6 & 11 & 19 & 24 & 32 & 37 & 43 & 9 & 17 & - & 6 & 14 & - \\
\hline
\end{tabular}

\section{References}

1. Abdelaziz FB, Masmoudi M (2012) A multiobjective stochastic program for hospital bed planning. J Oper Res Soc 63:530-538

2. Ahmadzad-Asl M, Abtahi H, Moradpoor M, Mehrabani V, Mashaii N, Bidaki R (2010) Community based organizations (CBOs) as an appropriate tool for harm reduction and prevention of drug abuse: the report of an experience. Iranian J Psychiatry Clin Psychol 16 (in Persian)

3. Barr A, Dekker M, Fafchamps M (2015) The formation of community-based organizations: an analysis of a quasiexperiment in Zimbabwe. World Dev 66:131-153

4. Benneyan JC, Musdal H, Ceyhan ME, Shiner B, Watts BV (2012) Specialty care single and multi-period location-allocation models within the veterans health administration. Socio Econ Plan Sci 46:136-148

5. Cardoso T, Oliveira MD, Barbosa-Póvoa A, Nickel S (2016) Moving towards an equitable long-term care network: A multi-objective and multi-period planning approach. Omega 58:69-85

6. Charnes A, Cooper WW, Ferguson RO (1955) Optimal estimation of executive compensation by linear programming. Manage Sci $1: 138-151$

7. Chen LH, Tsai FC (2001) Fuzzy goal programming with different importance and priorities. Eur J Oper Res 133:548-556

8. Cooper WW, Seiford LM, Tone K (2007) Data envelopment analysis: a comprehensive text with models, applications, references and DEA-solver software. Springer Science \& Business Media

9. Davari M, Haycox A, Walley T (2012) The Iranian health insurance system; past experiences, present challenges and future strategies. Iranian J Public Health 41:1-9

10. Davari S, Kilic K, Ertek G (2015) Fuzzy bi-objective preventive health care network design. Health Care Manag Sci 18:303-317

11. Galvao RD, Acosta Espejo LG, Boffey B (2002) A hierarchical model for the location of perinatal facilities in the municipality of Rio de Janeiro. Eur J Oper Res 138:495-517

12. Galvao RD, Acosta Espejo LG, Boffey B, Yates D (2006) Load balancing and capacity constraints in a hierarchical location model. Eur J Oper Res 172:631-646

13. Graber-Naidich A, Carter MW, Vedat V (2015) Primary care network development: the regulator's perspective. J Oper Res Soc 66:1519-1532

14. Gunes ED, Yaman H, Cekyay B, Verter V (2012) Matching patient and physician preferences in designing a primary care facility network. J Oper Res Soc 65:1-14

15. Guo M, Li B, Zhang Z, Wu S, Song J (2013) Efficiency evaluation for allocating community based health services. Comput Ind Eng 65:395-401

16. Khodaparasti S, Maleki HR, Jahedi S, Bruni ME, Beraldi P, Conforti D (2015) Balancing Efficiency and Equity in locationallocation models with an application to strategic EMS design. Optim Lett 1-18

17. Kim DG, Kim YD (2013) A Lagrangian heuristic algorithm for a public health care facility location problem. Ann Oper Res 206:221-240

18. Klimberg RK, Ratick SJ (2008) Modeling data envelopment analysis (DEA) efficient location/allocation decisions. Comput Oper Res 35:457-474

19. Lankarani KB, Alavian SM, Peymani P (2013) Health in the Islamic Republic of Iran, challenges and progresses. Med J Islamic Republic of Iran 27:42-49

20. Mehrdad R (2009) Health system in Iran. Int Med Community J 52:69-73

21. Mestre AM, Oliveira MD, Barbosa-Povoa A (2012) Organizing hospitals into networks: a hierarchical and multiservice model to define location, supply and referrals in planned hospital systems. OR Spectr 34:319-348 
22. Mestre AM, Oliveira MD, Barbosa-Povoa AP (2015) Locationallocation approaches for hospital network planning under uncertainty. Eur J Oper Res 240:791-806

23. Mitropoulos P, Mitropoulos I, Giannikos I (2013) Combining DEA with location analysis for the effective consolidation of services in the health sector. Comput Oper Res 40:22412250

24. Mohammadi M, Dehbari S, Vahdani B (2014) Design of a biobjective reliable health care network with finite capacity queue under service covering uncertainty. Transport Res E-Log 72:15-41

25. Raisa A, Viana A (2010) Operations research in health care: a survey. Int Trans in Op Res 18:1-31

26. Rahimi F, Esmaeili M, Nouri A, Mahdavi A (2011) The investigation social capital with emphasize to non-governmental organization role's in prevention of drug dependency. Soc Psychol Res 1:45-62. (in Persian)

27. Sahin G, Sural H, Meral S (2007) Locational analysis for regionalization of Turkish Red Crescent blood services. Comput Oper Res 34:692-704

28. Shekarian E (2015) A novel application of the VIKOR method for investigating the effect of education on housing choice. Int J Oper Res 24(2):161-183

29. Smith HK, Harper PA, Potts CN, Thyle A (2009) Planning sustainable community health schemes in rural areas of developing countries. Eur J Oper Res 193:768-777

30. Smith HK, Harper PR, Potts CN (2013) Bicriteria efficiency/equity hierarchical location models for public service application. J Oper Res Soc 64:500-512

31. Song BD, Ko YD, Hwang H (2015) The design of capacitated facility networks for long term care service. Comput Ind Eng 89:177-185
32. Steiner MTA, Datta D, Neto PJS, Scarpin CT, Figueira JR (2015) Multi-objective optimization in partitioning the health care system of Parana State in Brazil. Omega 52:53-64

33. Steuer R (1986) Multiple criteria optimization: theory, computation, and application. Willy

34. Sun L, DePuy GW, Evans GW (2014) Multi-objective optimization models for patient allocation during a pandemicinfluenza outbreak. Comput Oper Res 51:350-359

35. Syam S, Cote MJ (2010) A location-allocation model for service providers with application to not-for-profit health care organizations. Omega 38:157-166

36. Syam S, Cote MJ (2012) A comprehensive location-allocation method for specialized health care services. Oper Res Health Care $1: 73-83$

37. Taghvaii M, Zarrabi A, Rahimi BM (2010) Investigating effective factors of crimes in various areas. J Soc Sci 48:1-4. (in Persian)

38. Tiwari RN, Dharmar S, Rao JR (1987) Fuzzy goal programming an additive method. Fuzzy Sets Syst 24:27-34

39. Verter V, Lapierre SD (2002) Location of preventive health care facilities. Ann Oper Res 110:123-132

40. Zeng QL, Li DD, Yang YB (2013) VIKOR method with enhanced accuracy for multiple criteria decision making in healthcare management. J Med Syst 37(2):1-9

41. Zhang Y, Berman O, Verter V (2012) The impact of client choice on preventive health care facility network design. OR Spectr 34:349-370

42. Zimmermann HJ (1978) Fuzzy programming and linear programming with several objective functions. Fuzzy Sets Syst 1:45-55

43. http://www.farsnews.com/newstext.php?nn=13931020000880 (in Persian) 